\title{
Facial Soft Tissue Dynamics Before and After Primary Lip Repair
}

\author{
Carroll-Ann E. Trotman, B.D.S., M.A., M.S., \\ Orthodontics, School of Dentistry, The University of North Carolina at Chapel Hill, Chapel Hill, \\ North Carolina \\ Julian Faraway, Ph.D. \\ University of Bath, Bath, United Kingdom \\ Renate Soltmann, D.D.S., M.S., \\ School of Dentistry \\ Terry Hartman, B.S., and \\ Dental Research, School of Dentistry \\ John van Aalst, M.D., M.A., F.A.C.S., F.A.A.P. \\ Division of Plastic and Reconstructive Surgery, The University of North Carolina at Chapel Hill, \\ Chapel Hill, North Carolina
}

\begin{abstract}
Objectives-(1) To collect three-dimensional, dynamic facial images from two groups of infants: one group born with cleft lip and palate slated to have a primary lip repair and a second, age-matched, noncleft control group. (2) To develop analyses to determine differences in facial movement between infants with cleft lip and/or palate and noncleft control infants and to determine changes in facial movement before and after primary lip repair.

Design-Longitudinal, prospective case-control study.

Setting-Facial Animation Laboratory at the University of North Carolina School of Dentistry.

Participants-Two groups of infants: one group with unrepaired cleft lip and/or palate slated to have primary lip repair $(n=15)$ and a second group of age-matched, noncleft controls $(n=15)$.

Interventions-Movement testing before and 4 months after primary lip repair in infants with cleft lip and/or palate and at similar time points in noncleft infants.

Main Outcome Measures-Seven measures of facial movement.

Results-The range of facial movements increased by $17 \%$ for all infants during the 4-month period. Compared with the noncleft group (1) infants with unilateral cleft lip and/or palate had $50 \%$ less nasolabial movement, and this difference did not change due to the lip repair; and (2) infants with unilateral or bilateral cleft lip and/or palate had 58\% and $118 \%$ greater lateral upper lip movement, respectively, and 3.67 and 3.56 times greater asymmetry of movement, respectively, before lip repair. The procedure almost entirely removed these problems.
\end{abstract}

Conclusions-Primary surgical lip repair decreased hypermobility and improved asymmetry of upper lip movement. 


\section{Keywords}

facial movements; infants; primary lip repair; soft tissue

Previous research has shown that for many patients born with a cleft of the lip and/or palate $(\mathrm{CL} / \mathrm{P})$, the initial surgical repair of the cleft lip does not result in an acceptable outcome. At times, neither the patients nor the surgeons tend to be pleased with the long-term result. Later in life, some patients report dissatisfaction with their surgical outcomes, especially for the nasolabial region and the profile, and many wish to have additional secondary operations (Marcusson, 2001). Also, there are indications that less successful surgeries impact patients' socioeconomic outcomes in the form of diminished income and educational accomplishments when compared with their noncleft counterparts (Ramstad et al., 1995; Trost et al., 2007). Given these residual impairments in circumoral movements and disfigurements due to the primary or initial lip repair, a focus of our research has been on the functional and form outcomes of lip revision surgery-lip revisions are performed to correct this persistent facial disability that may remain after the primary lip repair (Marsh, 1990). The findings from our research, however, suggest that in order to decrease the frequency of lip revision surgery, equal emphasis should be directed toward the outcomes of the primary or initial lip repair. Support for this emphasis is based on parent reports of various psychological problems among their children due to their facial appearance, and there are indications that the quality of the mother-infant interactions are affected (Hunt et al., 2007). The method proposed here may be used to assist the surgeon with achieving the most ideal outcome for the primary lip repair and, thus, limit the need for subsequent lip revision surgery - an outcome that would be beneficial to both the patient and surgeon. In essence, this study lays the groundwork for understanding how to quantify circumoral movement in babies born with $\mathrm{CL} / \mathrm{P}$ in a way that answers important questions related to the efficacy of the primary lip repair.

As part of our previous research, we have developed novel objective, quantitative, threedimensional (3D) image-based metrics of facial movement and form and clinically relevant analyses (Trotman et al., 2007). The analyses allow clinicians to objectively discriminate between normal and impaired facial movements, as well as normal and disfigured facial forms, in children and adolescents with repaired CL/P within an age range of 5 to 21 years. These metrics are intended as a supplement to surgeons' subjective assessments (Gross et al., 1996; Trotman and Faraway, 1998; Trotman et al., 1998a; Trotman et al., 1998b; Trotman et al., 2000; Weeden et al., 2001; Trotman et al., 2003; Trotman et al., 2005; Trotman et al., 2007; Trotman et al., 2010; Trotman, 2011). Specifically, systematic and carefully designed studies revealed that in patients with repaired CL/P, circumoral movements are impaired, and the direction of the impairment tends to be related to the specific movement or animation action that a patient performs - a finding that reflects the underlying muscle anatomy and surgical scarring (Trotman et al., 2007; Trotman et al., 2010; Trotman, 2011). Using methods developed in our laboratory (Trotman et al., 2005; Trotman et al., 2007; Trotman et al., 2010; Trotman, 2011), the degree of improvement and/ or further impairment due to revision surgery were visualized, and we were able to uncover unexpected factors that affect the dynamic facial aesthetics in patients with CL/P. For example, during certain animations (e.g., smiling), impaired movements in the upper lip were accompanied by lower lip compensatory movements as well as unusual, unaesthetic circumoral movements (Trotman et al., 2000; Trotman et al., 2005).

In this study, a goal was to extend the evaluation of facial function/movement that was developed and used in patients with CL/P who have lip revision surgery to babies $(<6$ months) born with CL/P and to refine the methodology for an infant population. The specific 
aims were as follows: (1) To develop analyses to determine differences in facial function/ movement between the infants with CL/P and the noncleft control infants; and (2) to determine changes in facial function/movement before and after primary lip repair.

The hypothesis to be tested was that infants with CL/P exhibit different facial movement from noncleft control infants; and the movement of the noncleft control infants changes with age, whereas the movement of the CL/P infants changes with both age and the effects of surgery."

\section{Method}

This was a prospective, case-control study in which two groups of infants were followed and tested longitudinally: one group with unrepaired CL/P slated to have primary lip repair and another group comprising age-matched, non-cleft controls. Both groups of participants attended for two visits during which movement tests were conducted. The infants with CL/P were tested immediately before (first visit) and 4 months after (second visit) primary lip repair, and the control group infants were tested at similar times and visits. The study sample demographics for the CL/P and noncleft groups are given in Table 1. Overall, the groups were balanced by sex; however, the CLP infants were, on average, a month younger at the first visit. In the CL/P group, there were nine white, three African American, one Hispanic, and three mixed-race infants. There were five infants with unilateral complete cleft lip and palate, four with bilateral complete cleft lip and palate, and six with unilateral cleft of the lip and alveolus. In the noncleft group, there were eight white, three African American, two Hispanic, and three mixed-race infants. One infant in the noncleft group could not return for the second visit within the 4-month time frame, and another noncleft infant was recruited to bring the total to 16 infants in the noncleft group, 15 of whom had longitudinal data. Also, of the 15 infants recruited in the CL/P group, one infant did not return for the second visit, resulting in a total sample of 14 patients with longitudinal data in the CL/P group.

\section{Selection Criteria}

The infants with CL/P had either a unilateral or bilateral unrepaired cleft defect. They were recruited from the Craniofacial Center at the University of North Carolina (UNC) according to the following inclusion and exclusion criteria.

1. Inclusion criteria were unrepaired complete unilateral or bilateral cleft lip with or without a cleft palate; parent willingness to participate in the study; and an age less than 6 months.

2. Exclusion criteria were previous facial soft tissue surgery; a diagnosis of a craniofacial anomaly other than cleft lip and palate; and a diagnosis of collagen vascular disease, systemic neurologic impairment, or any condition that affects the neuromuscular and vascular tissues.

The noncleft infants were recruited from the UNC School of Medicine and were selected from those children that presented for routine medical review.

1. Inclusion criteria were parent willingness to participate in the study; and an age less than 6 months.

2. Exclusion criteria were previous facial soft tissue surgery; and a diagnosis of collagen vascular disease, systemic neurologic impairment, or any condition that affects the neuromuscular and vascular tissues.

Once the parents of the infants were contacted and the research assistant explained the purpose and protocol of the study to the parent(s), informed consent was obtained and the infant was enrolled. Consent and HIPAA documents were approved by the UNC Biomedical 
Human Subjects Institutional Review Board, and no infant was excluded from participation on the basis of sex or race.

\section{Data Collection}

At each visit, in order to collect three-dimensional (3D) dynamic facial images, a videobased tracking system (Motion Analysis TM, Motion Analysis Corporation, Santa Rosa, CA) was used (Trotman et al., 2007). The system tracked the movement of 3-mm diameter, retro-reflective markers secured to specific facial landmarks using hypoallergenic adhesive tape (Fig. 1a through 1c; Table 2). For the infants, the landmark positions were based on positions identified previously by other studies (Green and Wilson, 2006) and the need to have as many markers with comparable landmark positions in the noncleft infants and infants with $\mathrm{CL} / \mathrm{P}$ - both unilateral and bilateral cleft lip. Given the size of the landmarks and the size of an infant's upper lip, we were limited to five landmarks on the upper lip (Fig. 1; landmarks No. 6 through 10). Also, to get information on the commissure movement, landmarks 5 and 11 were included (Fig. 1). The nasal and lower jaw movements were captured with landmarks 2 through 4 and 12, respectively (Fig. 1). Table 2 gives the positions of these landmarks and their identification.

The system first was calibrated. The capture volume in which the infant's head was located was calibrated using a frame in the shape of an $\mathrm{L}$. The $\mathrm{L}$ frame had four markers fixed to exact, known locations on the frame. Next, the research assistant waved a wand with precisely located markers throughout the capture volume. This process located the exact camera positions and accounted for any geometric distortions in the camera lenses and accurately measured the camera lens focal lengths (Cortex 3.0 Reference Manual, 2011). The error in specifying marker position within the system was determined previously (Trotman et al., 1998b; Trotman et al., 2000) and approximated $0.53 \pm 0.45 \mathrm{~mm}$. Then, the infant was positioned within the calibrated area and the cameras adjusted to focus on the face. To facilitate this positioning, the infant was placed in an infant car seat that loosely supporting the back of the neck and head in order to allow an unobstructed view of the face (Fig. 2).

Several types of facial movements then were recorded as follows: spontaneous facial movements (Green and Wilson, 2006; Wilson and Green, 2009); facial movements in response to the infant's mother's voice; and facial movements elicited in response to the taste of salt and sugar substances placed on the infant's tongue with a cotton swab. The 3D landmark (movement) data were captured at a rate of 60 frames per second for 5-minute periods. Simultaneously, a synchronized video camera recorded the facial movements. Then, the landmarks were identified and tracked for each 5-minute recording. Two research assistants reviewed the tracked data to identify and isolate groups of movements or "epochs" for each infant in response to the taste of sugar and salt and during spontaneous smiling, crying, and suckling. The assistants knew whether sugar or salt was given to the infant. The differentiation between crying and smiling was based on the association of facial crying with audible crying. Suckling was always associated with jaw movement. Thus, the assistants were in $100 \%$ agreement in their identification.

The total number of valid movement epochs obtained for the CL/P and noncleft control groups at each visit and the total number of valid movement epochs for each animation are given in Table 3. Movement data were considered invalid when landmark data were missing. Valid but not complete data were captured from all 30 infants. The salt and sugar movement epochs numbered more than 80 . The epochs for the first and second visits and the smile, cry, and suckling movements numbered more than 100. 
Generalized Procrustes analysis (Dryden and Mardia, 1998) was used to remove whole-head motion and scale out head size differences among the infants, and several 3D measurements of facial soft tissue movement that were based on the changes in distance between pairs of landmarks were developed. The naming of these interlandmark distances represented the general directions of movement of the landmarks on the infant's face (Fig. 3).

(1) Whole Facial Range of Movement-This measurement of movement was based on the movement of each landmark as follows. For each landmark, all pairwise distances between frames were calculated. The corresponding pairs were summed and the 95th percentile calculated to give the whole facial range of movement (WFR). The 95th percentile was preferred to the maximum to provide some robustness and because we are interested more in the extremes of motion. The problem with using the median over a recorded sequence is that the babies do not perform a movement (e.g., crying) at a constant rate, so the median would measure a variable mixture of change in shape due to the movement and movement intensity that would be hard to interpret.

(2) Mid-Upper Lip Range of Movement-For the mid-upper lip landmark (No. 8, mlip), all pairwise distances between frames were calculated and the 95th percentile distance computed to give the mid-upper lip range of movement (MULRM).

(3) Vertical Upper Lip Movement-For each frame of movement, the distances between the right alar (No. 2, ralar) and the right outer upper lip (No. 6, roulip) and between the left alar (No. 4, lalar) and left outer upper lip (No. 10, loulip) were computed. The right and left distances were averaged. Then, over each animation (e.g., cry, smile), the difference between the 95th and the fifth percentile for the averaged distances was scaled by the median distance to give a measure of the range of vertical upper lip movement (VULM; Fig. 3a).

(4) Lateral Upper Lip Movement-For each frame of movement, the distances between the right inner upper lip (No. 7, riulip) and mlip, and between the left inner upper lip (No. 9, liulip) and mlip were computed. These right and left distances were averaged. Then, the difference between the 95th and the fifth percentile for the distance was scaled by the median distance to give a measure of the range of lateral upper lip movement (LULM; Fig. 3B).

(5) Center Vertical Facial Movement-For each frame of movement, the distance between the nose bridge (No. 1, nbridge) and midchin (No. 12, chin) landmark was computed. Then, the difference between the 95th and the fifth percentile for the distance was scaled by the median distance to give a measure of the range of center vertical facial movement (CVFM; Fig. 3C).

(6) Nasolabial Movement-For each frame of movement, the distance between the nose tip (No. 3, ntip) and mlip was computed. Then, the difference between the 95th and the fifth percentile for the distance was scaled by the median distance to give a measure of the range of nasolabial movement (NLM; Fig. 3D).

(7) Asymmetric Movement: Asym (Figure 3E)—For each frame of movement, the differences between the riulip and mlip distance and between the liulip and mlip were computed. The measure represented the range of this difference (without the median scaling, which was not appropriate here) and was a measure of the asymmetric movement (ASYM; Fig. 3E). 
For all seven 3D measures, the data were averaged over replicates. For some combinations of visit, subject, and animation, no valid replicates were observed; however, there were on average 1.9 observations per combination.

\section{Statistical Analyses}

For each 3D measure (1 through 7), a model of the following form was applied:

$$
\log \left(m_{i}\right)=\mu+a_{(i)}+v_{(i)}+c_{(i)}+v c_{(i)}+\varepsilon_{i},
$$

where $m$ is the measure; $a$ is the animation, which takes five possible levels: sugar, salt, smile, cry, suckling; $v$ is the visit, first or second; and $c$ is the group, which can be unilateral $\mathrm{CL} / \mathrm{P}(\mathrm{UCL} / \mathrm{P})$, bilateral CL/P (BCL/P) or noncleft. The subscript (i) denotes the corresponding level of that factor in the $i$ th row of the data.

Other models that included animation by cleft type, animation by visit, and also three-way interactions were computed. Only a visit by group interaction was included in these models because other interactions were found to be not statistically significant.

\section{Results}

Table 4 provides the results for the model. It gives the effect of each factor and significant interaction factors on the different measures of facial movement along with the levels of significance. The factor reference levels were cry, first visit, and control group. Thus, each of the stimulated movements in response to sugar and salt as well as the spontaneous behaviors of smile and suck were measured as the difference from cry. The visit factor represented the difference between the second visit and the first visit. The UCL/P and BCL/ $\mathrm{P}$ factors represented the difference between the UCL/P and BCL/P infants and the controls, respectively. The UCL/P/Control/Visit and $\mathrm{BCL} / \mathrm{P} / \mathrm{Control} / \mathrm{Visit}$ factors were interaction factors. Also, from the data in the Table 4 and the model, several other results can be calculated.

The results show that there was a significant visit effect for the WFR. Specifically, at the second visit, which was at the end of the 4-month period of time, the WFR increased by the exponent of 0.16 , or 1.17 , representing a $17 \%$ increase on average over all the infants. There was a significant effect for infants with UCL/P with the NLM. These infants demonstrated $50 \%$ less NLM (exponenting $-0.69=1.50$ or $50 \%$ decrease) when compared with the noncleft controls, and this difference did not change as a result of the lip repair.

For the LULM, the UCL/P and BCL/P infants had 58\% and 118\% greater movement, respectively, than the controls at the first visit. These results were marginally significant. At the second visit after the lip repair, this difference with the controls was essentially removed. This calculation at the second visit is based on the following mean measures in Table 4 (first row for measure LULM). Using the model, $\log (\mathrm{UCL} / \mathrm{P})=$ control value of $0.07+\mathrm{UCL} / \mathrm{P}$ value of $0.46+\mathrm{UCL} / \mathrm{P}$ interaction value of $-0.41=0.12$. The value of 0.12 then was exponentiated to give 1.13, which represented a $13 \%$ increase for LULM at the second visit. The same calculations for the infants with BCL/P shows a 5\% increase in LULM at the second visit. Similarly, for the ASYM, the UCL/P and BCL/P participants were found to have 3.67 (267\%) and 3.56 (256\%) times more asymmetric movement, respectively, than the controls at the first visit. After the lip repair and at the second visit, this level of asymmetry was reduced to $2.10(110 \%)$ and $1.62(62 \%)$ times the controls for the UCL/P and BCL/P infants, respectively. Thus, for the LULM, the surgery brought the CL/P infants very close to the normal; whereas, for the ASYM, those with CL/P were closer to normal. 
There were several significant differences among the animation types-the spontaneous animations and those animations that were stimulated by the taste substances. Because the factor reference levels were cry, first visit, and control group, the data in Table 4 would permit other calculations to be made. For example, the predicted value of $\log (\mathrm{WFR})$ for a cry in the control group for the first visit is 3.27 , but for a smile in the UCL/P group on the second visit it is $3.27-0.14+0.16+0.05-0.03=3.31$. In general, relative to the cry, the results were that the VULM was $36 \%$ less with salt, $45 \%$ less during the smile, and $14 \%$ less during suckling. The LULM demonstrated $57 \%$ and $52 \%$ greater movement with sugar and salt, respectively. The CVFM demonstrated 36\% greater movement with sugar and during suckling, $46 \%$ greater movement with salt, but $19 \%$ less movement during the smile. From a subjective perspective, when observing the infants' responses to the sugar and salt taste stimuli, most responded with suckling and a grimace and, to a lesser extent, chewing.

\section{Discussion}

This study focused on outcomes of facial function or movement as a result of primary lip repair in infants with unrepaired CL/P. Based on the study hypothesis, it was demonstrated that infants with unrepaired CL/P show differences in facial soft tissue movements when compared with noncleft infants, and the effects of age and lip repair change the soft tissue movements. The study findings provide knowledge of the functional capabilities of the facial soft tissue of the cleft lip, which would be of value to surgeons so that they may be better informed about the biological effects of the cleft on facial soft tissue dynamics. Moreover, it might be expected that this information could allow surgeons to more critically evaluate the outcomes of surgery in order to improve the soft tissue function and limit the need for additional revision surgeries. Previous researchers have used the Baby Facial Action Coding System (FACS) to evaluate facial muscle function/movement (Ekman and Friesen, 1978). Although the Baby FACS is more sensitive than a simple visual subjective evaluation, the Baby FACS still relies on observer ratings of movements of specific facial units or regions seen on videotapes - an approach that is based on rating scales and requires the calibration of raters. The method used in this study produces quantitative movement data for the evaluation of circumoral function/movement in adolescents and infants and can be used to supplement surgeons' subjective appraisal of a patient's facial movement (Trotman et al., 2010; Trotman, 2011). It is interesting that other researchers have successfully used this quantitative approach to study the development of behavioral and circumoral movements during speech in noncleft infants (Green and Wilson, 2006; Wilson and Green, 2009).

The finding that the range of facial movements increased on average over all infants during the 4-month period was expected. It is known that newborn infants have limited and basic facial expressive behaviors related to early survival instincts (e.g., breathing) and that targeted and deliberate facial expressions appear to develop with age, and in part, in response to parental/caregivers' facial expressions (Darwin, 1872). This early lack of animated movements limits the measurements of facial movement in newborn infants to basic behaviors such as crying, suckling, and responses to stimulation of taste and smell. In this study, the taste stimuli of sugar and salt were used to successfully elicit facial animations, but only the sugar stimulus appeared to be specific to lateral lip movement. From direct observation of the infants, we found that the taste stimuli resulted mainly in suckling and grimace-like movements followed by chewing movements. Past research has shown that in comparison with odor stimuli, taste stimuli elicit reliable and predictable movements in infants (Armstrong et al., 2007), and infants distinctively discriminate based on sweet, bitter, and sour tastes (Ganchrow et al., 1983; Armstrong et al., 2007). For example, Soussignan et al. (1997) demonstrated that responses to odor stimuli varied significantly with few stimulus-dependent facial responses. Other researchers have shown 
that infants around 2 months of age show a wide range of responses to, and do not seem to discriminate, salt stimuli (Stein et al., 2006). In future studies, the stimuli will include a wide range of tastes - sweet, sour, salty, and bitter substances. One caveat with the use of taste stimuli in infants with unrepaired CL/P is that the palatal taste buds may be limited or missing, and even after the palate repair, taste buds on the palate may be decreased compared with noncleft infants; thus, taste responses may be limited or impaired for infants with CL/P.

Before the primary lip repair, we found that infants with CL/P had much greater upper lip movement and greater asymmetry of movement compared with the noncleft infants. One reason for this finding is that in the infant with a cleft lip, the orbicularis oris muscle is disrupted. In the case of a unilateral cleft lip, the upper lip is separated into two parts or segments; whereas, for a bilateral cleft lip, the muscle is separated into three segments. As a result, the large muscles of the cheek and the levator exert a greater influence and pull on the lateral upper lip segments, resulting in exaggerated movements as well as uncontrolled asymmetry of movement. As expected, this asymmetry is decreased after the lip repair, no doubt as a result of both the repair and early scarring at 4 months. For visual examples of infants who demonstrate these findings, see the Web site http://people.bath.ac.uk/jjf23/face/ baby/. It is tempting in patients with a unilateral cleft lip to use the noncleft side of the lip as a comparison for the cleft side. Although such a comparison might be applicable to compare the asymmetry in lip form at rest, our past research (Trotman et al., 2003) has shown that it is unwise to use this comparison to assess lip asymmetry during movements. The reasons include the following: (1) Our research has shown that objective measures generated from the entire upper lip region provided the best overall reliability; (2) the differences in measurements between the cleft side of the upper lip and the noncleft side are small, and (3) the impairment in movement due to a lip repair or a lip revision would likely affect the movement of the noncleft side of the upper lip to varying degrees that depend on numerous factors such as the cleft location, the extent of the cleft, skill of the surgeon, and effects of scarring (Trotman et al., 2003).

In summary, this study has demonstrated that infants born with CL/P exhibit a hypermobility of the upper lip segments and asymmetry of facial soft tissue movements. In the short term-over a 4-month period-primary lip repair greatly decreased the hypermobility and improved the asymmetry. However, a 4-month period of follow-up represents a very limited time span, and generally, a minimum of 12 months is needed to allow sufficient time for healing, scar remodeling, and tissue softening. In future studies, we expect to extend this timeline for follow-up and to determine whether surgeons can incorporate this method in the treatment planning of patients with CL/P to improve the soft tissue surgical outcomes in terms of dynamic animated movements during emotive behaviors and spontaneous behaviors related to speech development.

\section{Acknowledgments}

This study was supported by grants R01 DE13814-01A1 \& 3R01DE013814-08S1 from the National Institute of Dental and Craniofacial Research (NIDCR) to Dr. Carroll-Ann Trotman.

\section{References}

Armstrong JE, Hutchinson I, Laing DG, Jinks AL. Facial electromyography: responses of children to odor and taste stimuli. Chem Senses. 2007; 32(6):611-621. [PubMed: 17510090]

Cortex 3.0 Reference Manual. Motion Analysis Corporation; Santa Rosa, CA: 2011.

Darwin, C. The Expression of the Emotions in Man and Animals. John Murray; London: 1872.

Dryden, L.; Mardia, KV. Statistical Shape Analysis. Wiley; Chichester: 1998. 
Ekman, P.; Friesen, WV. The Facial Action Coding System (FACS): A Technique for the Measurement of Facial Action. Consulting Psychologists Press; Palo Alto, CA: 1978.

Ganchrow JR, Steiner JE, Daher M. Neonatal facial expressions in response to different qualities and intensities of gustatory stimuli. Infant Behav Dev. 1983; 6:189-200.

Green JR, Wilson EM. Spontaneous facial motility in infancy: a 3D kinematic analysis. Dev Psychobiol. 2006; 48:16-28. [PubMed: 16381029]

Gross MM, Trotman CA, Moffatt KS. A comparison of three-dimensional and two-dimensional analyses of facial motion. Angle Orthod. 1996; 66:189-194. [PubMed: 8805913]

Hunt O, Burden D, Hepper P, Stevenson M, Johnston C. Parent reports of the psychosocial functioning of children with cleft lip and/or palate. Cleft Palate Craniofac J. 2007; 44:304-311. [PubMed: 17477751]

Marcusson A. Adult patients with treated complete cleft lip and palate. Methodological and clinical studies. Swed Dent J Suppl. 2001; 145:1-57. [PubMed: 11400538]

Marsh JL. When is enough enough? Secondary surgery for cleft lip and palate patients. Clin Plast Surg. 1990; 17:37-47. [PubMed: 2302918]

Ramstad T, Ottem E, Shaw WC. Psychosocial adjustment in Norwegian adults who have undergone standardised treatment of complete cleft lip and palate. I. Education, employment and marriage. Scand J Plast Reconstr Hand Surg. 1995; 29:251-257.

Soussignan R, Schaal B, Marlier L, Jiang T. Facial and autonomic responses to biological and artificial olfactory stimuli in human neonates: re-examining early hedonic discrimination of odors. Physiol Behav. 1997; 62:745-758. [PubMed: 9284493]

Stein LJ, Cowart BJ, Beauchamp GK. Salty taste acceptance by infants and young children is related to birth weight: longitudinal analysis of infants within the normal birth weight range. Eur J Clin Nutr. 2006; 60:272-279. [PubMed: 16306932]

Trost O, Gradel J, Danino A, Malka G. Socioeconomic adjustment in young cleft lip-palate patients. Rev Stomatol Chir Maxillofac. 2007; 108:383-387. [PubMed: 17681569]

Trotman CA. Faces in 4D-why do we care and why the 4th dimension? Am J Orthod Dentofacial Orthop. 2011; 140:895-899. [PubMed: 22133956]

Trotman CA, Faraway JJ. Sensitivity of a method for the analysis of facial mobility: II. Inter-landmark separation. Cleft Palate Craniofac J. 1998; 35:142-153. [PubMed: 9527311]

Trotman CA, Faraway JJ, Essick GK. 3-D nasolabial displacement during movement in cleft lip and palate patients. Plast Reconstr Surg. 2000; 105:1273-1283. [PubMed: 10744215]

Trotman CA, Faraway JJ, Losken HW, van Aalst J. Functional outcomes of cleft lip surgery. Part II: quantification of nasolabial movement. Cleft Palate Craniofac J. 2007; 44:607-616. [PubMed: 18177193]

Trotman CA, Faraway JJ, Phillips C. Visual and statistical modeling of facial movement in patients with cleft lip. Cleft Palate Craniofac J. 2005; 42:245-254. [PubMed: 15865457]

Trotman CA, Faraway JJ, Phillips C, van Aalst J. Effects of lip revision surgery in cleft lip/palate patients. J Dent Res. 2010; 89(7):728-732. [PubMed: 20439935]

Trotman CA, Faraway JJ, Silvester KT, Greenlee G, Johnston LE Jr. Sensitivity of a method for the analysis of facial mobility: I. Vector of displacement. Cleft Palate Craniofac J. 1998ba; 35:132141. [PubMed: 9527310]

Trotman CA, Phillips C, Essick GK, Faraway JJ, Barlow SM, Losken HW, van Aalst J, Rogers L. Functional outcomes of cleft lip surgery. Part I: study design and surgeon ratings of lip disability and the need for lip revision. Cleft Palate Craniofac J. 2007; 44:598-606. [PubMed: 18177192]

Trotman CA, Phillips C, Faraway JJ, Ritter K. Association between subjective and objective measures of lip form and function: an exploratory analysis. Cleft Palate Craniofac J. 2003; 40:241-248. [PubMed: 12733951]

Trotman CA, Stohler CS, Johnston LE Jr. Measurement of facial soft tissue mobility in man. Cleft Palate Craniofac J. 1998b; 35:16-25. [PubMed: 9482219]

Weeden JC, Trotman CA, Faraway JJ. Three-dimensional analysis of facial movement in normal adults: influence of sex and facial shape. Angle Orthod. 2001; 71:132-140. [PubMed: 11302590] 
Wilson EM, Green JR. The development of jaw motion for mastication. Early Hum Dev. 2009; 85:303-311. [PubMed: 19185434] 


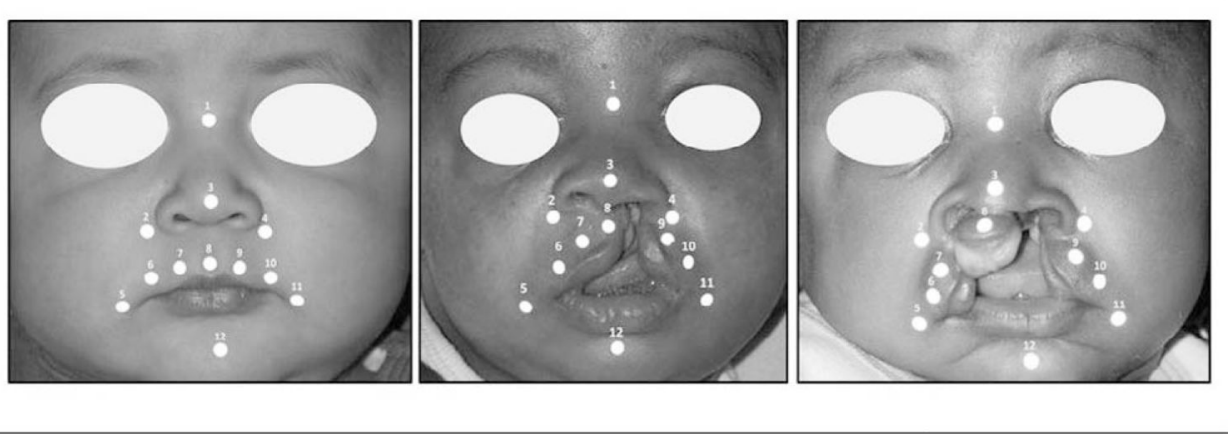

FIGURE 1.

Landmark locations. a: a noncleft infant, b: infant with unilateral CL/P, and c: infant with bilateral CL/P. 


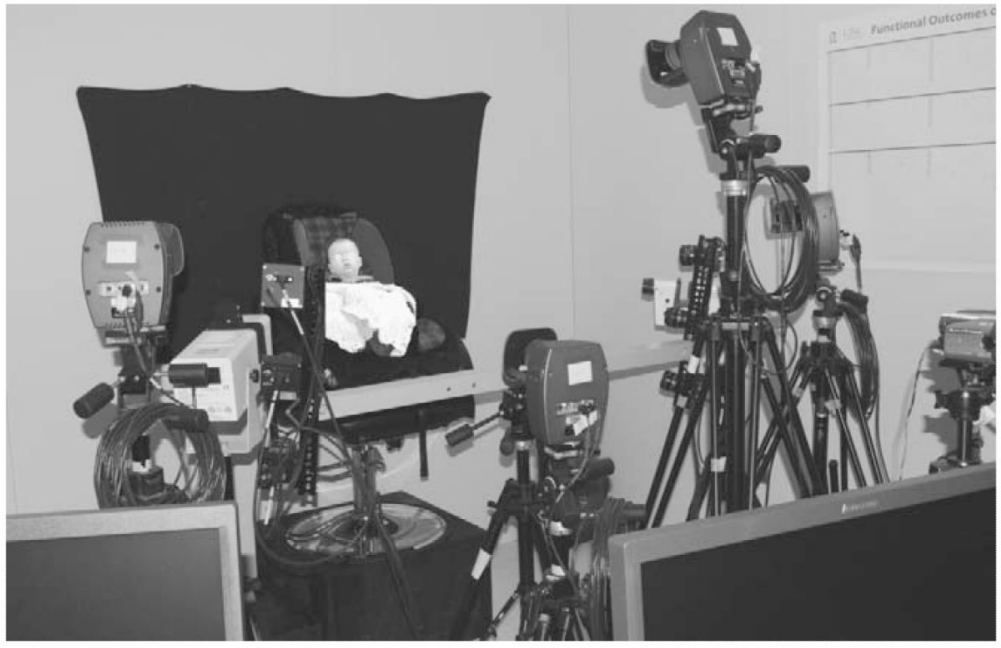

FIGURE 2.

System configuration. 

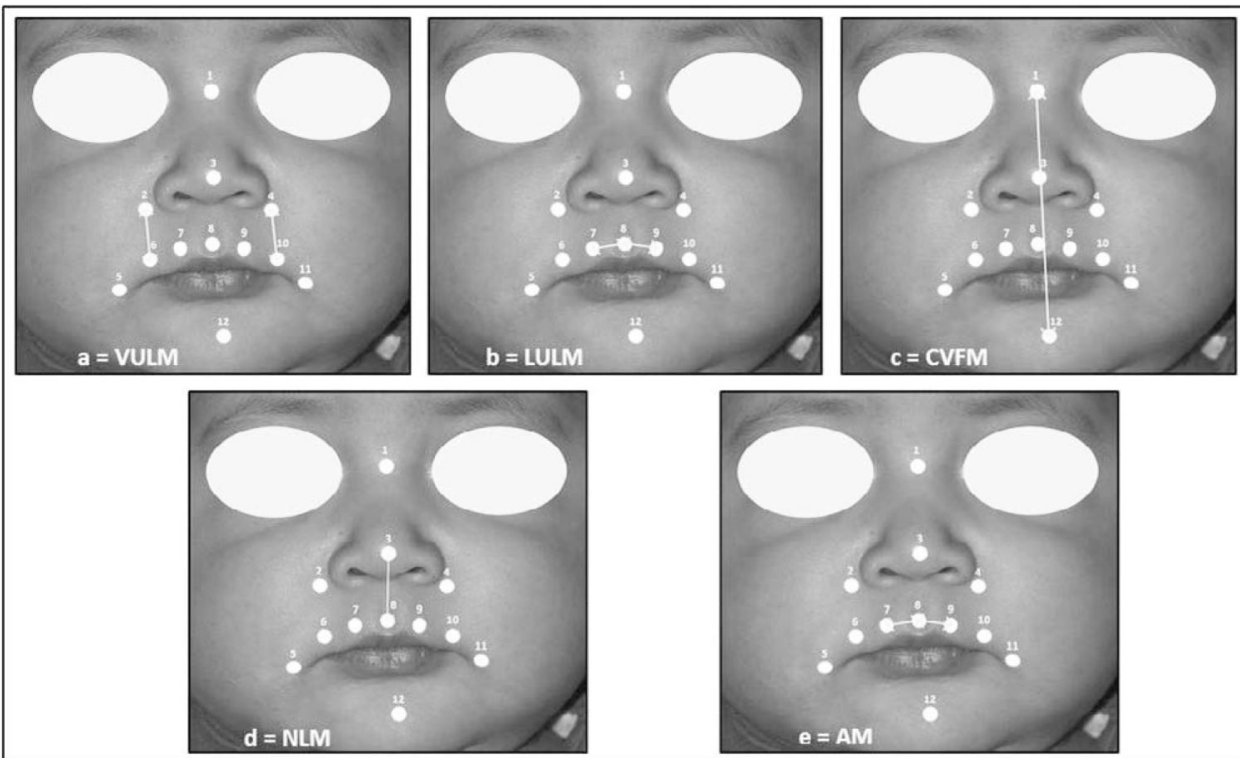

FIGURE 3.

Illustration of measures of distance between landmarks: a: VULM, b: LULM, c: CVFM, d: NLM, e: AM. 
TABLE 2

\section{Landmark Locations and Identification}

\begin{tabular}{lll}
\hline Landmark & \multicolumn{1}{c}{ Name } & Identification \\
\hline 1 & Nose bridge & Point midway between left and right inner canthi \\
2 & Right alar & Point on the base of the nostril lateral to the right alar \\
3 & Nose tip & The most prominent position on the midpoint of the nostril tip \\
4 & Left alar & Point on the base of the nostril lateral to the left alar \\
5 & Right commissure & Point just lateral to the right corner of the mouth \\
6 & Right outer upper lip & Point just above the upper vermillion at one third the distance between (5) and (8) with an origin from (5). \\
7 & Right inner upper lip & Point just above the upper vermillion at one third the distance between (5) and (8) with an origin at (8) \\
8 & Mid-upper lip & Point just above the upper vermillion and below the columella. \\
9 & Left inner upper lip & Point just above the upper vermillion at one third the distance between (8) and (11) with an origin at (8) \\
10 & Left outer upper lip & Point just above the upper vermillion at one third the distance between (8) and (11) with an origin at (11) \\
11 & Left commissure & Point just lateral to the left corner of the mouth \\
12 & Midlip (chin) & Point on the midline of the chin \\
\hline
\end{tabular}


TABLE 3

Number of Valid Movements

\begin{tabular}{lc}
\hline & No. \\
\hline Visit & \\
Visit 1 CL/P* & 125 \\
Visit 1 control & 147 \\
Visit 2 CL/P & 138 \\
Visit 2 control & 179 \\
Animation & \\
Cry & 161 \\
Smile & 138 \\
Suckling & 120 \\
Salt & 83 \\
Sugar & 87 \\
\hline
\end{tabular}

${ }^{*} \mathrm{CL} / \mathrm{P}=$ cleft lip and/or palate. 


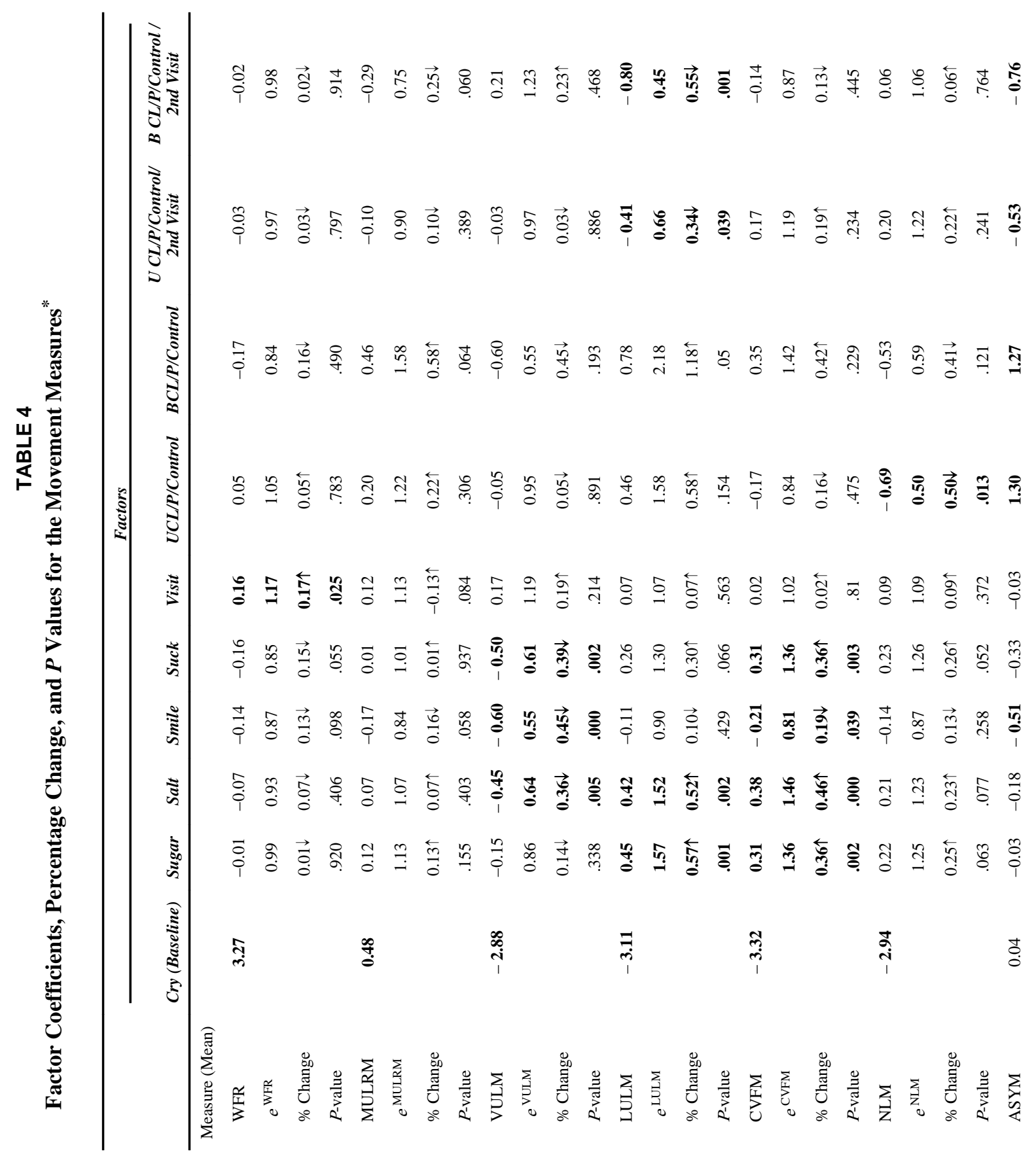




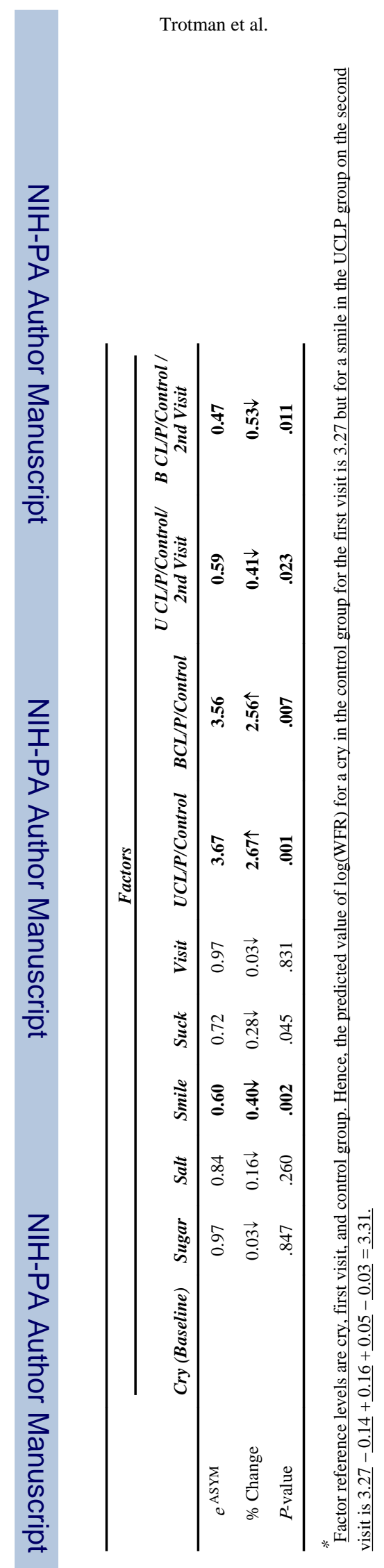

Page 18

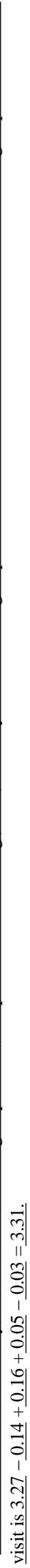

